Original Research Article

\title{
Effect of steroid on platelet count of dengue patients admitted at Sapthagiri Institute of Medical Sciences and Research Centre: a retrospective study
}

\author{
Piyali Hazra*, Navya Teja K., L. Padma
}

Department of Pharmacology, Sapthagiri Institute of Medical Sciences and Research Centre, Bangalore, Karnataka, India

Received: 16 September 2019 Accepted: 17 October 2019

*Correspondence to:

Dr. Piyali Hazra,

Email:piyals.16@gmail.com

Copyright: (C) the author(s), publisher and licensee Medip Academy. This is an openaccess article distributed under the terms of the Creative Commons Attribution NonCommercial License, which permits unrestricted noncommercial use, distribution, and reproduction in any medium, provided the original work is properly cited.

\begin{abstract}
Background: New treatment strategies are attempted to treat the fatal complications of the dengue infection, as significant numbers of adult and children are affected resulting in considerable economic impact. Corticosteroids in various regimens have been used in the last 2 decades empirically on the basis of immunomodulation or treat the vascular leakage that occurs in severe dengue.

Methods: A descriptive study was conducted among 60 patients who were dengue positive and received treatment from the Department of Medicine at SIMSRC during the period of June-August 2018. The study group (30 patient) was given treatment with intravenous dexamethasone $8 \mathrm{mg}$ initially, followed by $4 \mathrm{mg}$ TID for 3 days. The control group (30 patient) received only IV fluids and antipyretics. The rise in the mean platelet counts over three days was analyzed in both groups were analyzed.

Results: The results of this study revealed that after 10-12 hours of initiating steroids in the study group, a higher and early rise of platelet count was achieved. There was gradual improvement and increase in platelets in 3 days' duration in all 30 of the patients. The mean platelet count (the primary outcome) in the study group was significant compared with the control group.

Conclusions: At present, realistic approaches for the early intervention and proper treatment protocol are required to be developed to prevent high mortality and morbidity due to dengue. The drawback of this study was, it was not a double-blind placebo-controlled trial and there were less number of patients.
\end{abstract}

Keywords: Dengue, Dexamethasone, Low dose steroid, Mean platelet count

\section{INTRODUCTION}

The dengue infection is a mosquito-borne viral disease caused by flavivirus with four distinct serotypes (DENV$1,2,3,4)$. The infections are spread among humans by mosquitoes Aedes aegypti and Aedes albopictus. ${ }^{1}$

Globally the burden of dengue infection all over is estimated to be 390 million of which 96 million of these manifest clinically. ${ }^{2}$ The incidence of dengue epidemics following seasonal climatic change occur during each rainy season. Many are affected during epidemics, though most patients suffer a simple febrile illness, a small but significant proportion go on to develop the dengue shock state, with associated fatalities. The case fatality rate of the more severe infections is $1 \%$ or higher, particularly in children and young adults.

Clinical manifestations of symptoms occur after inoculation period of the virus, around 4-7 days. At the beginning presented as an acute febrile illness, characterized by high temperature, malaise, retro-orbital headache, myalgia, backache, nausea, loss of appetite, and vomiting. The hallmark is capillary leakage in severe 
dengue that occurs from third to seventh day of illness. The symptomatic phase of dengue for management purposes, is divided into three phases: the febrile phase, the critical phase, and the recovery phase. ${ }^{1}$ During the febrile phase, patients are generally hemodynamically stable; dehydration can occur due to severe vomiting. Platelet counts decline, leukopenia is a feature, and varying degrees of haemoconcentration can occur. In severe dengue platelet count drops drastically below $100 \times 109 / 1$. However low platelet counts often do not result in overt haemorrhage. It is capillary leakage that gives rise to serious complications. ${ }^{4}$

The pathogenesis of dengue fever has not been clearly understood. Thrombocytopenia is a constant manifestation of dengue fever that often leads to severe dengue haemorrhagic fever and dengue shock syndrome. ${ }^{11}$ There are studies that points, severe manifestations of dengue having an immunological basis rather than being due to a direct tissue damage by the virus. ${ }^{6}$

Recent evidence suggests that transient disruption of the surface glycocalyx, lining the vascular endothelium, takes place. The cytokines tumor necrosis factor alpha, interleukin IL-2, IL-6, IL-8, IL-10, IL-12, and interferon gamma are significantly elevated in severe dengue when compared with uncomplicated dengue fever. ${ }^{6,7}$ Most evidence suggest that vascular endothelial cell dysfunction is induced by cytokine and chemical mediators, which is an important factor leading to plasma leakage. $^{3}$

The possible mechanism of thrombocytopenia in dengue haemorrhagic fever has been strongly suspected due to the increased peripheral destruction of the antibody coated platelets. ${ }^{9,10}$ The other modes include acute bone marrow suppression which leads to a megakaryocytic condition and enhanced platelet destruction by the reticuloendothelial system.

According to WHO guidelines the management of dengue is largely supportive, with careful fluid replacement. ${ }^{1}$ However due to lack of specific treatment, an effective vaccine, and difficulties in vector control physician in the South East Asian countries clinicians use corticosteroids empirically, based on the immunological basis for stabilizing the capillary permeability and complication of dengue. $^{3}$

New treatment strategies are attempted to treat the fatal complications of the dengue infection, as significant numbers of adult and children are affected resulting in considerable economic impact. ${ }^{4}$ Corticosteroids in various regimens have been used in the last 2 decades empirically on the basis of immunomodulation or treat the vascular leakage that occurs in severe dengue. ${ }^{4}$ So this study is planned to study the efficacy of steroid on platelet count of dengue patients.
The objective was to assess the efficacy of intravenous dexamethasone (Inj. Dexamethasone $8 \mathrm{mg}$ initially followed by $4 \mathrm{mg}$ IV TDS for 3 days) on the mean platelet count during the acute stage of dengue fever with thrombocytopenia in comparison to dengue patients who have not received intravenous dexamethasone.

\section{METHODS}

This study was conducted at Medicine Department of Sapthagiri Institute of Medical Sciences and Research Center, Bangalore. All the patients who were confirmed serologically to be NS1, IgM positive cases of dengue and received treatment from the department of medicine in SIMS and RC were included. Patients of either gender above 18 years were included. The patients with concomitant disease like malaria, Enteric fever, leptospirosis etc. were excluded. The study design is descriptive study on secondary data following universal sampling method by which the data collection was conducted. The study period was for 2 months (JuneAugust 2018). Ethical clearance was obtained from the institutional ethics committee before the commencement of the study. Confidentiality was assured throughout the study.

The data of all patients who were admitted and received treatment for dengue was analyzed. All the necessary information like the demographic data (age, gender, etc.), clinical history, investigation (haemoglobin, PCV, platelet count on day 1 , day 2, day 3) and treatment regimen (steroid, other medication) were recorded in the structured patient proforma. These data thus collected from the case files maintained in the medical record section of the hospital were analyzed.

\section{Statistical analysis}

The data was statistically analyzed using Microsoft Excel expressed in frequencies, standard deviation as appropriate. The rise in the mean platelet counts over three days, was calculated by the independent student $\mathrm{t}$ test in both groups and $\mathrm{p}$ value less than 0.05 was considered significant.

\section{RESULTS}

The 60 patients of this study, satisfying the inclusion criteria were given routine treatment with Intravenous dexamethasone $8 \mathrm{mg}$ initially, followed by $4 \mathrm{mg}$ every 8 hours, thereafter for 3 days and IV fluids were given as and when required. The control group received only IV fluids and antipyretics as necessary. The monitoring of the platelet count, temperature, pulse, BP, weight, fluid intake output chart, edema, haematocrit value, blood glucose and electrolytes was done for daily three days of the study period.

The mean rise in the platelet count was measured daily in both the groups, which was taken as the primary 
outcome. The demographic and the haematological features of both the groups were comparable, as shown in (Table 1). There were no significant differences in the base line characters like the mean (SD) age, sex and $\mathrm{Hb} \%$. The mean rise in the platelet counts over three days, which was calculated by the independent sample $t$ test in the both groups, is shown in (Table 2).

The mean rise in the platelet counts over three days, which was calculated by the independent student " $t$ " test in the both groups, is shown in (Figure 1).

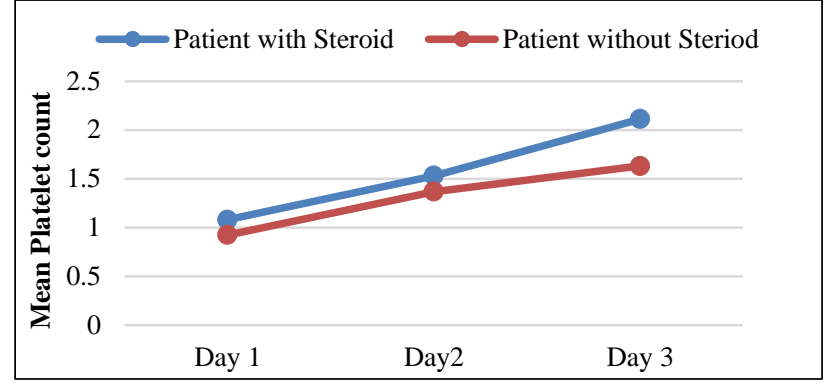

Figure 1: Mean rise in platelet count over 3 days between study and control group.

Table 1: Demographic comparison between study group and control group.

\begin{tabular}{|c|c|c|c|c|}
\hline \multirow{3}{*}{ Sex } & Characteristics & Study group & Control group & $P$ value \\
\hline & Male & $17(56.7)$ & $18(60)$ & \multirow{2}{*}{0.679} \\
\hline & Female & $13(43.3)$ & $12(40)$ & \\
\hline Age & Mean Age \pm Sd & $28.17 \pm 13.82$ & $28.03 \pm 10.83$ & 0.967 \\
\hline Hemoglobin & Mean $\mathrm{Hb} \pm \mathrm{Sd}$ & $13.93 \pm 2.27$ & $13.45 \pm 2.74$ & 0.45 \\
\hline
\end{tabular}

Table 2: Mean platelet counts of control and study group over days and results of independent student " $t$ " test.

\begin{tabular}{|llllll|}
\hline \multirow{2}{*}{ Droup } & & $\mathbf{N}$ & Mean & Std. deviation & P value \\
& Study group & 30 & 1.0793 & 0.52313 & 0.228 \\
\cline { 2 - 5 } Day 2 & Control group & 30 & 0.9421 & 0.31958 & 0.22 \\
\cline { 2 - 5 } & Study group & 30 & 1.5303 & 0.41864 & 0.44510 \\
\hline \multirow{2}{*}{ Day 3} & Control group & 30 & 1.3938 & 0.001 \\
\cline { 2 - 6 } & Study group & 30 & 2.0950 & 0.47511 & 0.46763 \\
\hline
\end{tabular}

\section{DISCUSSION}

The results of this study revealed that, after 10-12 hours of initiating steroids in the study group, a higher and early rise of platelet count was achieved. There was gradual improvement and increase in platelet count over 3 days' duration in all 30 of the patients. The mean platelet count (the primary outcome) in the study group was significant compared with the control group. All patients later followed up in OPD showed level of more than $1,00,000$ platelet count.

None of the patients had any bleeding complications or went into coagulopathy and MODS. The mortality rate was nil. Age and sex had no influence in the outcome of treatment. One patient showed drop till 3500 without bleeding manifestations after initiating steroids but on $2^{\text {nd }}$ day showed gradual improvement and reaching 75,000 in 4 days of intravenous steroids. Similar results were elicited in the study conducted by Ather Pasha et al. ${ }^{8}$

The use of low dose corticosteroids helps in stopping the ongoing immune destruction of platelets and hence avoiding platelet transfusion and its associated complications and overall its cost effectiveness in the management of viral thrombocytopenia in Indian scenario.
The results of this study are in concordance with the conclusions which were opined by other studies conducted by Futrakul et al, showed a positive response with a high dose of methyl prednisolone with severe DSS which was unresponsive to fluid replacement. ${ }^{5}$

\section{CONCLUSION}

In conclusion this study revealed low dose regimen, dexamethasone was effective in achieving a higher and early rise of platelet count in dengue fever preventing serious complications. There were several limitations in this study. Sample size was smaller than that required to show a moderate difference. At present, realistic approaches for the early intervention and proper treatment protocol are required to be developed to prevent high mortality and morbidity due to dengue.

Limitations of this study was, it was not a double-blind placebo controlled trial and there were less number of subjects.

\section{ACKNOWLEDGEMENTS}

The authors would like to thank the faculty of department of general medicine, Sapthagiri Institute of Medical 
Sciences and Research Centre, Bangalore, for their cooperation and guidance in data collection.

Funding: No funding sources

Conflict of interest: None declared

Ethical approval: The study was approved by the Institutional Ethics Committee

\section{REFERENCES}

1. World Health Organization, Dengue: guidelines for diagnosis, treatment, prevention and control. Geneva, Switzerland: World Health Organization; 2009.

2. Kularatne SA. Survey on the management of dengue infection in Sri Lanka: opinions of physicians and pediatricians. South Asia J Trop Medi Pub Health. 2005;36(5):1198.

3. Panpanich R, Sornchai P, Kanjanaratanakorn K. Corticosteroids for treating dengue shock syndrome. Cochr Data Syst Rev. 2006(3).

4. Stasi R, Provan D. Management of immune thrombocytopenic purpura in adults. In Mayo Clin Pro. 2004;79(4):504-22.

5. Futrakul P, Poshyachinda MA, Mitrakul $\mathrm{CH}$, Kwakpetoon SM, Unchumchoke PO. Hemodynamic response to high-dose methyl prednisolone and mannitol in severe dengue-shock patients unresponsive to fluid replacement. South Asian J Trop Medi Pub Health. 1987;18(3):373-9.
6. Chappell D, Jacob M, Hofmann-Kiefer K, Bruegger $\mathrm{D}$, Rehm M, Conzen $\mathrm{P}$, et al. Hydrocortisone preserves the vascular barrier by protecting the endothelial glycocalyx. J Am Soci Anesthesiol. 2007;107(5):776-84.

7. Shashidhara KC, Murthy KS, Gowdappa HB, Bhograj A. Effect of high dose of steroid on plateletcount in acute stage of dengue fever with thrombocytopenia. J Clini Dia Res. 2013;7(7):1397.

8. Pasha A, Ahmed SB. Role of low dose hydrocortisone in the management of viral thrombocytopenia. Int $\mathrm{J}$ Pharma Medi Biol Sci. 2014;3(3):95.

9. Halstead SB. Antibody, macrophages, dengue virus infection, shock, and hemorrhage: a pathogenetic cascade. Rev Infect Dis. 1989;11(4):S830-9.

10. Green S, Vaughn DW, Kalayanarooj S, Nimmannitya S, Suntayakorn S, Nisalak A, et al. Early immune activation in acute dengue illness is related to development of plasma leakage and disease severity. J Infect Dis. 1999;179(4):755-62.

Cite this article as: Hazra P, Teja KN, Padma L. Effect of steroid on platelet count of dengue patients admitted at Sapthagiri Institute of Medical Sciences and Research Centre: a retrospective study. Int J Basic Clin Pharmacol 2019;8:2681-4. 